\title{
Téoros
}

Revue de recherche en tourisme

\section{Image et perception de Montréal}

\section{Louis St-Jean et Émile Vogel}

Volume 10, numéro 3, novembre 1991

Destination Montréal : d’hier à demain

URI : https://id.erudit.org/iderudit/1079205ar

DOI : https://doi.org/10.7202/1079205ar

Aller au sommaire du numéro

Éditeur(s)

Université du Québec à Montréal

ISSN

0712-8657 (imprimé)

1923-2705 (numérique)

Découvrir la revue

Citer cet article

St-Jean, L. \& Vogel, É. (1991). Image et perception de Montréal. Téoros, 10(3), 51-57. https://doi.org/10.7202/1079205ar d'utilisation que vous pouvez consulter en ligne.

https://apropos.erudit.org/fr/usagers/politique-dutilisation/ 


\section{Louis St-Jean et Émile Vogel de Montréal}

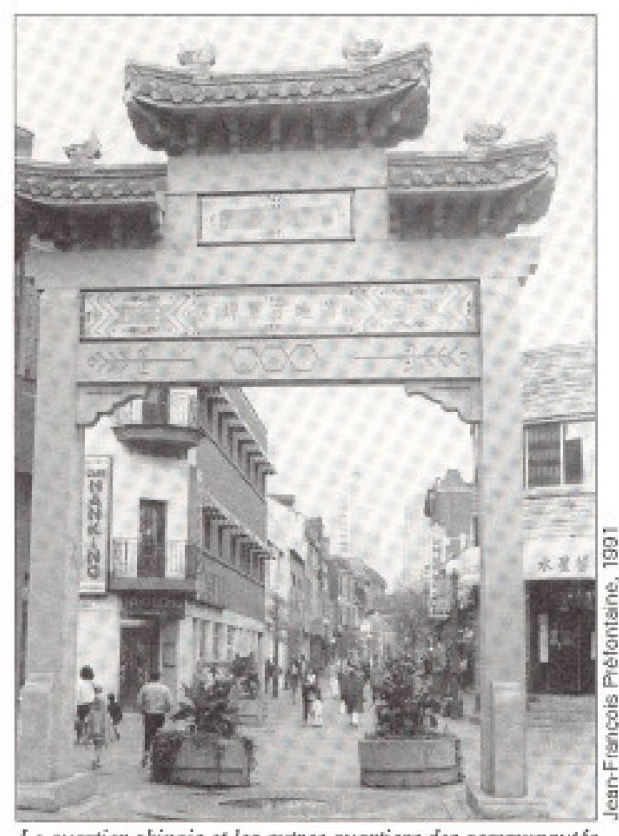

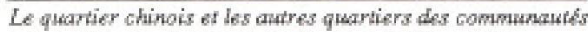
culturelles sont-ils arsocis a limage do Montral?

Lareprésentationmentale quel'on se fait d'un être ou d'une chose constitue son image. Montréal, en tant que produit de consommation, possède son image.

Pour les spécialistes de la mise en marché, 1 "image que projette un produit (une destination) revêt une importancecapitale. Eneffet, celle-ci déterminera le positionnement dudit produit, c'est-à-dire la perception qu'ont les consommateurs des attributs de celui-ci et sa situation par rapport à ses concurrents.

Dès qu'un acheteur (touriste) potentiel entre en contact avec un produit, le processus de formation d'image commence. Le contact peut se faire de différentes façons. D'une part, les consommateurs prennentconscience et se familiarisent avec les caractéristiques d'unedestination par lecourant d' information qu'ils reçoivent de diverses sources. Publicitế, promotion, agences de voyage, parents, amis, associations de consommateurs, etc.,

Louis St-Jean est delégué commercial a l'Office des congress et du tourisme du Grand Montréal et Émile Vogel est consultant en tourisme. sont des réseaux d'information permettant à un individu de se faire une image d'une destination. D'autre part, le contact direct avec la destination permet au touriste de confirmer(ou infirmer)etde préciser l'image qu'il a de celle-ci. Laperception de Montréal en sera une directement rẹliée à son évaluation du patrimoine touristique de la ville. Il faut donc être conscient que notre image s'améliore ou se détériore moment après moment et jour après jour en fonction de la somme totale des expériences que les touristes vivent au contact de notre ville.

Dans la majorité des cas, les touristes (visiteurs ou non) voient dans une destination donnée bien plus qu' un simple ensemble de caractéristiques physiqueset objectives. Les touristes perçoivent les destinations avec des significations, des attributs et des valeurs symboliques plus ou moins différents. Pour certains, Montréal c'est bien plus que le Vieux-Montréal et le Stade olympique, c'est aussi une ville au caractère vivant et dynamique où la joie de vivre est à 1 'honneur et la sécurité omniprésente.

Cette description de la ville ne reflète cependant pas la perception exacte qu'en ont tous les touristes. En effet, pour un visiteur américain, Montréal est perçue comme étant une ville très sécuritaire et propre alors que pour les touristes Québécois, ces deux attributs ont moins d'importance.

Les différents segments de clientèles développentdonc desperceptionscommunes mais aussi spéciliques de Montréal selon leurs besoins, leurs intếrêts, leurs expériences antérieures et leurs environnements.

En fait, les groupes homogènes de touristes (visiteurs ou non) ne perçoiventpas le produit (Montrếal) de la même façon parce que chacun a tendance à ne percevoir que les attributs qui sont importants pour lui. L'identification (done la projection) de ces attributs est essentielle puisque c'est à partir de ceux-ci que les touristes vont sans doute comparer les diverses destinations qui leurs sont offertes avant d'en choisir une.
Ajoutons que même si tous les visiteurs considéraient le même ensemble d'attributs, la valeur du produit Montréal serait probablement perçue différemment par chacun des segments de marchés. En effet, ces derniers $n^{*}$ accordent pas la même importance à chacun des attributs d'un produit. Ainsi, pour certains, la séceurité peut être un facteur déterminant dans le choix (ou non) d'une destination. Pour d'autres, même en sachant que ladite destination estréputée non sécuritaire, d'autres attributs jugés plus importants lui feront opter pour celle-ci.

Bien comprendre les mécanismes de la perception est un impératif majeur pour les intervenants touristiques. Cela est necessaire au développement d'une bonne stratégie de gestion de la perception. Ils doivent présenter le produit Montréal selon les dimensions perceptibles propres à chaque groupe de visiteurs en insistant sur les plus importants.

\section{L'image de Montréal}

A l'aide des quelques études disponibles et en prenant connaissance des commentaires émis par plusieurs intervenants du domaine touristique, il est possible de dresser une image globale du Montréal touristique: Montréal est une ville internationale, ville d'ambiance à caractère européen et francophone possédant une architecture intéressante comprenant un réseau souterrain impressionnant.

Viennent s'annexer à ces éléments la joie de vivre et l'hospitalité de ses habitants. Tout cela en plus de dégager une sensation de sécurité dans une ville propre.

\section{Ville internationale}

Son caractère international, Montréal le doit aux grands événements tels la tenue de $1^{4}$ Exposition universelle de 1967, des Jeux olympiques d'été de 1976 ainsi qu*a la présentation des Floralies en 1980.

Par la même occasion, Montréal a pu se faire connaittre et répandre sa réputation en terme de destination touristique. Plusieurs autres 
attractions ou événements spéciaux ont, au fil des ans, formées l'image de Montréal.

En effet. Montréal est reconnue comme étant l'hôte de grands événements sportifs à caractère international. Nous n'avons qu'à penser au Marathon International de Montréal, au Challenge Player's Ltée, au Grand Prix Molson du Canada, et depuis peu au Grand Prix des Amériques (cyclisme).

Toutes ces activités ont contribué à donner à Montréal une réputation de ville à caractère international et dynamique. Cette image internationale différencie Montréal des autres villes canadiennes. À titre d'exemple, mentionnons que lors de la commémoration du 50e anniversaire du «Radio City Music Halls à New York, 50 villes à travers le monde furent invitées et la seule ville canadienne sollicitée fut Montréal.

La métropole francophone a su se créer une place parmi les grandes villes internationales comme en témoigne la présence de nombreux sièges sociaux d'organismes internationaux. L'OACI, l'IATA et la SITA n'en sont que quelques exemples.

\section{Ville francophone et culturelle}

Montréal jouit également d'un élément culturel qui la distingue des autres grandes villes nord-américaines. Onparle icidufailfrançais de la ville, celui-là même qui incite les journalistes à présenter Montréal comme étant le Paris de l'Amérique du Nord.

Cette particularité est perçue par les touristes non-européens comme étant une possibilité degoûter aux charmes d'une ville européenne francophone sans pour autant avoir à se déplacer sur le vieux continent. Par exemple, le touriste américain semble percevoir Montréal comme étant une destination où il peut vivre un certain dépaysement tout en sentant l'omniprésence d'un certain standard nordaméricain.

La ville possède une originalité culturelle fondée d'abord sur son caractère francophone mais aussi influencée par la présence anglophone et les groupes multi-ethniques.

En considérant que la culture est un élément essentiel pour attirer les touristes, il ne fait aucun doute que Montréal possède une force d'attraction bien particulière.

\section{Ville ambiance}

Montréal possède également une image de ville d'ambiance. Contrairement à une ville où les seuls attraits majeurs seraient les monuments, les musées et autres sites physiques, Montréal est reconnue pour l'ambiance qu'elle dégage.

Ses boutiques, ses restaurants, ses terrasses, l'aspect Quartier Latin de la rue St-Denis, son nightlife, le Vieux-Montréal et depuis peu la rue St-Laurent sont tous des éléments qui, une fois consommés par le visiteur, tendentà lui faire ressentir l'ambiance propre à Montreal.

L'humour, le jazz, les films voire les feux d'artifice font également de Montréal une ville reconnue comme très active dans le réseau des grands festivals internationaux où 1'ambiance est toujours au rendez-vous. Une ambiance qui constitueà elle seule un attrait. Un attrait que l'on peut aujourd'hui considérer comme étant sacralisé. Sacralisation dont les originessemblentremonteràl'époque d'Expo '67.

\section{Ville architecturale}

L'image touristique de Montréal est aussi celle d'une ville possédant une architecture du 2le siècle qui n'a rien à envier au gigantisme urbain américain. Néanmoins, elleasu sauvegarder son histoire à travers ses vieux bâtiments.

Montréal c'est aussi la plus grande ville souterraine au monde permettant l'accès à tous les services et ce, toujours a l'abri des intempéries. À ce niveau, la ville jouit d'une image unique.

\section{Perception de Montréal}

Définir l'image de Montrếal en tant que destination touristique telle que perçu par ses différents marchés-cibles est une tâche complexe puisqu'il n'existe que très peu de données précises à ce sujet.

Nous allons tout de même tenter de dégager les perceptions qu'ont les clientèles touristiques traditionnelles de Montréal que sont lesQuébécois, les Américains, les Canadiens des autres provinces et les visiteurs d'outremer. Ces segments représentent respectivement $40 \%, 25 \%, 25 \%$ et $5 \%$ des visiteurs de la métropole.

\section{Les perceptions des Américains}

Pour cerner la perception qu'ont les Américains (visiteurs ou non) de Montréal, quatre documents ont particulièrement retenu notre attention. Il s'agit de l'étude du comportement et de l'attitude des différents segments de clientèles touristiques de Montréal (Secor, 1989), del'étude de perception des Américains originaires de Boston et de New York par rapport au Québec comme destination de vacances (Impact Recherche, 1990), de l'étude sur le marché Américain des voyages d'agrément, 1989 (Longwoods Research Group, 1991) et du rapport d'analyse des coupures de la Presse américaine (OCTGM, 1991).

Voici donc ce qui ressort de ces études:

On retrouve dans l'étude de Secor de 1989, une évaluation de Montréal par les touristes interviewés en vertud'une grillede 24 critères. Pour chacun des critères, le touriste était invité à formuler son appréciation de Montréal, chiffré de 1 à 9 ( 9 étant une excellente evaluation). Le tableau 1 fait état de l'évaluation de Montréal par les touristes Américains.

Globalement, la perception qu'ont les touristes Américains de la ville est très bonne. $\mathrm{La}$ propreté, la qualité de l'hébergement et des restaurants, la sécurité, le magasinage, l'accueil, la culture française, 1 'efficacité du transport local, l'attrait généralde la villeet le côté fêttard de la ville sont particulièrement appréciés.

À l'opposé, le coût de l'hébergement et de la restaurationet la température sont leséléments les moins bien évalués par cette clientèle.

L'un des objectifs de l'étude d'Impact recherche de 1990 était de déterminer les forces et les faiblesses du Québec et de Montréalen tant que destination touristique des Américains.

L'analyse des résultats nous permet de constater que les aspects les plus appréciés de Montréal sont: la beauté de la ville, les restaurants, la vie nocturne, l'hospitalité et la propreté (cf. tableau 2). À l'opposé, leséléments négatifs sont le rude service - the atittude of people, la temperature et la langue française.

Ainsi, si l'attitude des Montréalais est considérće comme un élément négatif par certains Américains, une partie plus importante deceux-ciconsidèrentl'hospitalitéetl'accueil 


\section{TABLEAU 1}

Mesures attitudinales des Américains face à Montréal

Les critères pour lesquels on observe une moyenne de plus de 7,5 peuvent être considérés comme des axes de force de Montréal.
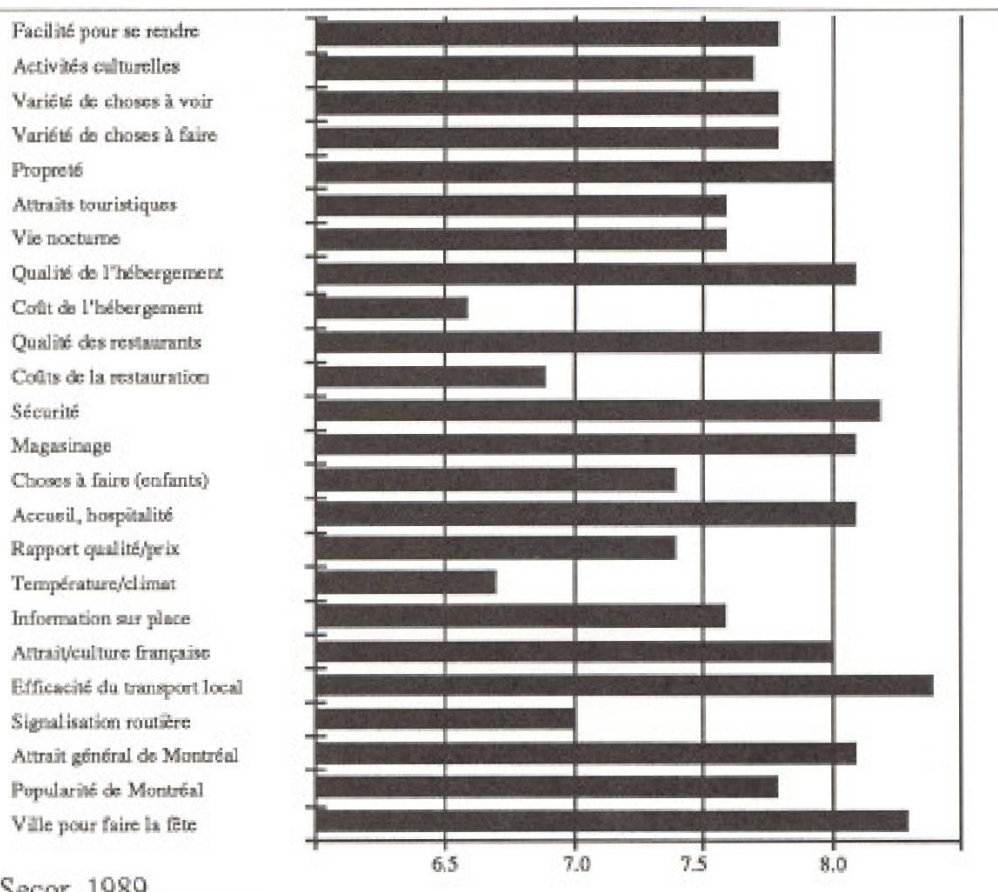

Source: Secor, 1989.

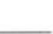

chaleureux comme une force de Montréal. Il existe donc des sentiments partagés à ce sujet.

D'après une évaluation de certains éléments spécifiques à notre ville en tant qu'attrait touristique, il ressort que la vie nocturne, la variếté des activités, la qualité de l'hébergement et des restaurants et l'hospitalité sont les éléments qui furent évalués le plus favorablement par les rếpondants. Le ski et le coût de l'hébergement et des restaurants sont les aspects qui reçoivent les résultats les moins élevés (cf. tableau 3 ).

Le tableau 3 présente aussi la perception de l'évaluation des différentes composantes touristiques de Montréal par les non-visiteurs.

Les événements culturels sont sous-évalués par les non-visiteurs par rapport aux visiteurs, de même que la variété des activités, là vie nocturne et les magasins. Le coût des hébergementsetrestaurants est surévaluépar lenon-visiteur. Lesaspects lesmieux évalués parcesdemiers furent l'hospitalité et la qualité des hébergements et restaurants.
Il est à noter que $60 \%$ à $70 \%$ des personnes interrogées ont été incapables d'évaluer les différents attraits pour cause d'ignorance à leur sujet.

Également, cette étude nous révèle que malgré le fait que le Québec souffre d'une perception socio-politique véhiculant une attitude négative à l'égard des anglophones en gếnéral,unnombre trèsminimed'Américains seront influencés négativement par ce contexte par rapport à une visite éventuelle dans notre province (Montréal).

Même s'il faut interpréter certains des résultats de l'étude de Longwood de 1991 avec prudence, ceux-ci nous donnent tout demême certaines indications concernant la perception qu'ont les Américains de la ville.

Montréal semble jouir dune image générale très favorable à l'égard des touristes Américains. En effet, pour ces demiers, Montréal vient au second rang pami les villes nordaméricaines qu'ils aimeraient vraiment visiter (cf, tableau 4).

Toujours selon cette étude, les villes du Canada (dont Montréal) sont jugées belles, ci- vilisées, propres, sûres, présentant un charme étranger que n'ont pas les villes américaines.

Par rapport à ces demières, les villes canadiennes sont perçues comme étant peu animées, sans beaucoup de vie noctume, manquant de magasins, de bons restaurants et d'hôtels, etoffrantpeu d'attractions culturelles.

De plus, même si les vacances urbaines au Canada sont jugées un peu plus chères, elles n'en sont pas moins considérées commeétant d'une excellente valeur. La possibilité de découvrir des attractions urbaines dans un milieu ếtranger, pas tellement loin de chez soi, est sans doute l'une des raisons pour lesquelles les villes canadiennes offrent encore un très bon rapport qualité/prix.

Cependant, la perception que se font les Américains des prix au Canada a tendance à être de plus en plus négative. Cela est attribuable à la force du dollar canadien par rapport a la devise américaine.

Enfin, il semblerait que les Américains qui visitent les grandes villes canadiennes ont une expérience touristique qui dépasse de beaucoup leurs attentes.

L'analyse des coupures de presse américaine nous donne des renseignements sur l'image que projette Montréal à travers les divers quotidiens Américains. Cela nous renseigne aussi sur les perceptions qu'ont les joumalistes americains de notre ville. Resumons les thèmes les plus souvent relevés:

- Le leitmotiv de nombreux articles est Visit France without leaving the continent. Montréal est aussi souvent comparée à Paris et on la présente comme étant la deuxième plus grande ville françaiseau monde après cette dernière.

- La majorité des articles font référence au fait français. Les Montréalais sont perçus comme des hôtes majoritairement francophones mais qui se débrouillent très bien en anglais et ce, lorsqu'ils ne sont pas entièrement bilingues. Ils sont également perçus comme très accueillants et tolérants envers les visiteurs provenantdes ÉtatsUnis. Dans plusieurs articles cependant, on mentionne que les Montréalais sont moins tolérants envers les anglophones du Québec et du Canada en général. 
- On fait souvent mention de la Jote de vive et de laccuell chaleurewx des Montréalais et du caractêre vivant et dynamique de la ville.

- Lecaractèremultiethnique de Montréal est souvent exprimé par la présentation des restaurants d'origines ethniques différentes. La qualité de la restauration revient également dans plusieurs articles. Le commentaire général peut se résumerainsi; đà Montréal, on mange de tout, on mange bien et ce, à tous les prixo.

- La rue St-Laurent, haut lieu de la nouvelle culture et dumulticulturalisme est de plus en plus citée dans la presse américaine. Elle sert de prétexte à plusieurs articles sur la ville.

- Montréal est encore perçue comme une ville sécuritaire et propre. On la compare très avantageusement aux grandes villes américaines. Le système de transport en commun - et particulièrement le métro de Montréal - reçoivent egalement les mêmes commentaires.

- LeMontréal souterrain est maintes fois cité comme un elément qui distingue Montréal. Il est perçu à la fois comme une attraction et comme un équipement qui permetet incite les touristesa visiter Montréal à l'abri des intempéries hivernales.

- Montréal est Également perçue comme une ville où $l$ architecture nouvelle et ancienne se marient avantageusement, cequiluiconfere beaucoup d'intérêt. À cet égard, on remarque que le Centre canadien d'architecture occupe une place de plus en plus importante dans la Presse américaine.

Les opinions émises par les journalistes américains sont très positives, ce qui n'est guère étonnant de la part de chroniqueurs touristiques.

Une analyse des éléments communs à toutes ces recherches nous permet de constater les faits suivants:

- La plupart des Américains perçoivent Montrél comme étant une belle ville (par sonarchitecture, entre autrechose); une ville sécuritaire et propre où la qualité de l'hébergement et de la restauration est excellente.
TABLEAU 2

Éléments les plus appréciés à Montréal (en \%)

The beauty of its

Les restaurants

Le wnightlife*

L'hospitalité

La proprete

Variété - activités

Atmosphère française

Le sshopping"

Différence culturelle

Le côté historique

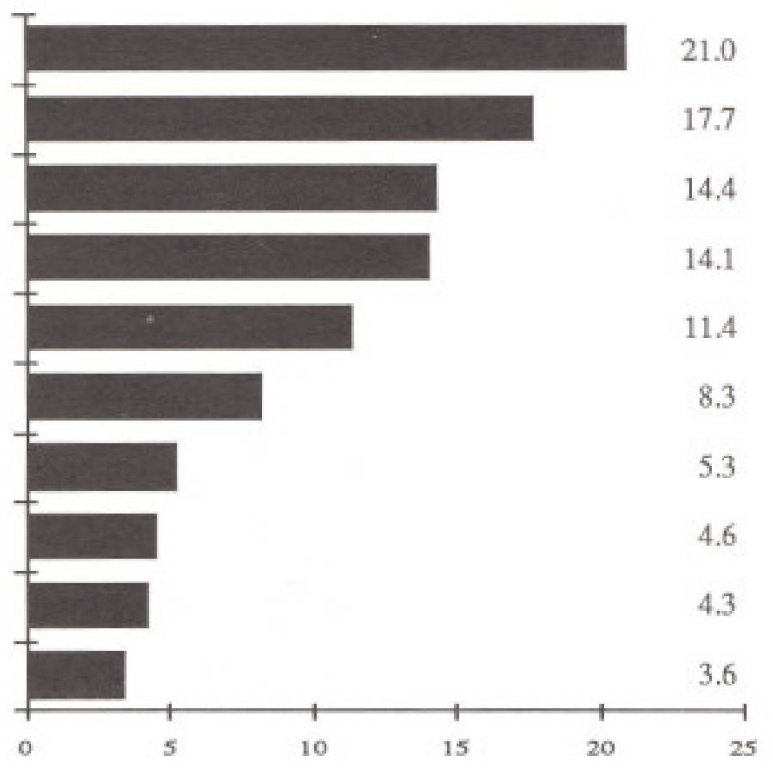

Source: Impact Recherche..

TABLEAU 3

Evaluation comparative par les visiteurs et non-visiteurs

Événements culturels

Variete activites

Sites historiques

Nightlifew et divertissements

Qualité des hôtels

Coût des hôtels

shopping:

Ski

Hospitalité

Attrait de cul. fran.

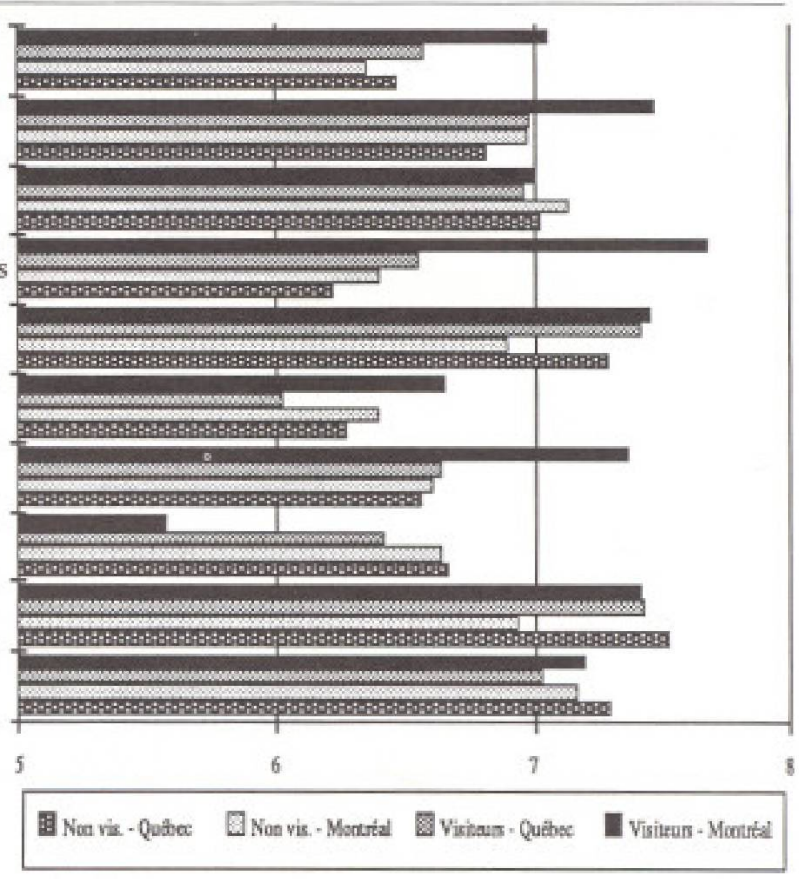

Source: IMPACT Recherche.

N.B.: Évaluation sur une échelle de 1 a 9 (9 étant le plus élevé). 
- Le fait français est reconnu par tous mais pas toujours apprécié. Il semblerait que les touristes américains veuillentêttre submergés de culture française quand ils viennent à Montréal mais être servis en anglais.

En général, la perception que se font les Américains de notre accueil est bonne. De plus, Montrálal est perçue comme offrant une vie nocturne intéressante.

Finalement, tous les Américains perçoivent Montréal comme étant une ville dispendieuse où la température y est incertaine.

Pour les trois prochains groupes-cibles, seule la perception qu'ont les visiteurs à l'égard de Montréalestanalysce (surtout grâceăl'étude Secor 1989) puisqu'il n'existe pas d'étude valable nous renseignant sur la perception des non-visiteurs.

\section{Les perceptions des Québécois}

Les Québécois représentent une part importante de l'ensemble de la clientèle traditionnelle de Montréal. Les raisons de leurs passagesà Montréal se traduisent principalement par la visite de parents ou d'amis ou dans le but d'assister à un événement sportif ou culturel.

Dans l'ensemble, les Québécois perçoivent Montréal comme étant une ville intéressante pourlesactivités detoutes sortes qu'ils peuvent y retrouver (évếnements culturelsousportifs, le magasinage, le vie nocturne). La qualité des restaurants et de l'hébergement est bien perçue tandis que les coûts qu impliquentces établissements le sont beaucoup moins (cf. tableau 5).

Évidemment, pour les Québếcois, l'attrait culture française de la ville ne les touche que très peu et ne constitue pas une force d'attraction en soi. L'acceuil et l'hospitalité que dégage Montréal à leur égard sont plutôtt perçus négativement. Deplus, les Québécois qui sont en visite à Montréal perçoivent souvent la ville comme étant malpropre et non sécuritaire. La mauvaise perception de ces éléments constitue un paradoxe face à celle desautresclientèles de la ville concernantces aspects.
TABLEAU 4

Pourcentage de touristes urbains américains qui conviennent que les villes suivantes sont un endroit qu'ils aimeraient vraiment visiter (en \%)
1) San Francisco
2) Montréal
3) Washington, D.C.
4) Nouvelle-Orléans
5) Québec
6) Vancouver
7) Totontó
8) Boston
9) Denver
10) Charleston
11) Calgary
12) New York
13) Los Angeles
14) Atlanta
15) Chicago
16) Ottawa
17) Saint-Louis
18) Winnipeg
19) Edmonton
20) Halifax
21) Indianapolis

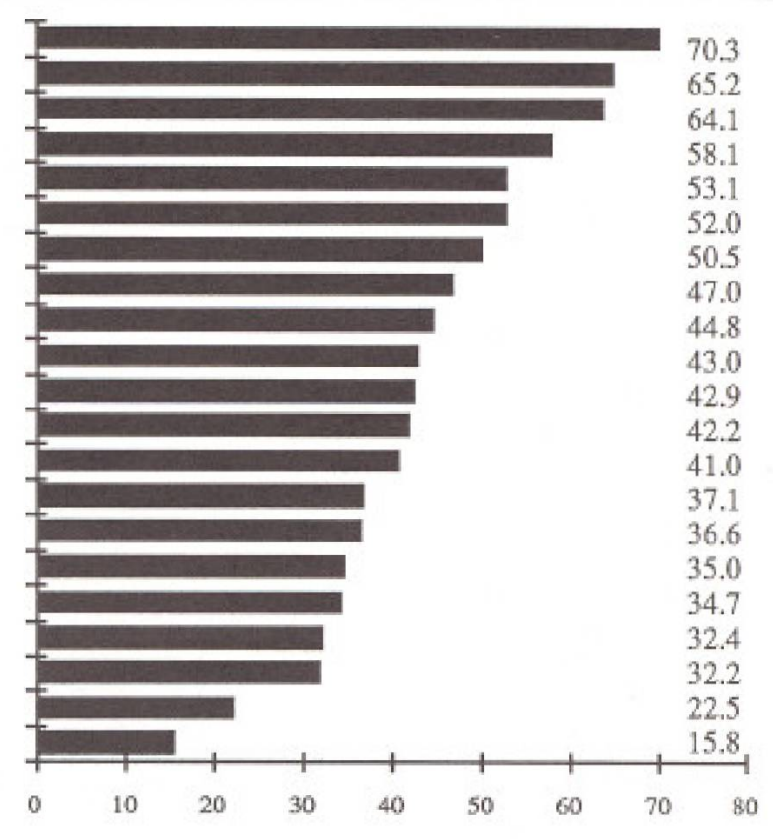

( $n=400$ )

Source: Longwood Research Group 1991.

\section{TABLEAU 5}

Mesures attitudinales des Québécols face à Montréal

Les critères pour lesquels on observe une moyenne de plus de 7,5 peuvent être considérés comme des axes de force de Montréal.

Facilité pour se rendre Activités culturelles Variété de choses à voir Variété de choses à faire Propreté

Attraits touristiques

Vie nocturne

Qualité de l'hébergement

Coût de l'hébergement

Qualité des restaurants

Coûts de la restauration

Sécurité

Magasinage

Choses à faire (enfants)

Accueil, hospitalité

Rapport qualité/prix

Temperature/climat

Information sur place

Attrait/culture française

Efficacité du transport local

Signalisation routičre

Attrait général de Montréal

Popularité de Montréal

Ville pour faire la fête

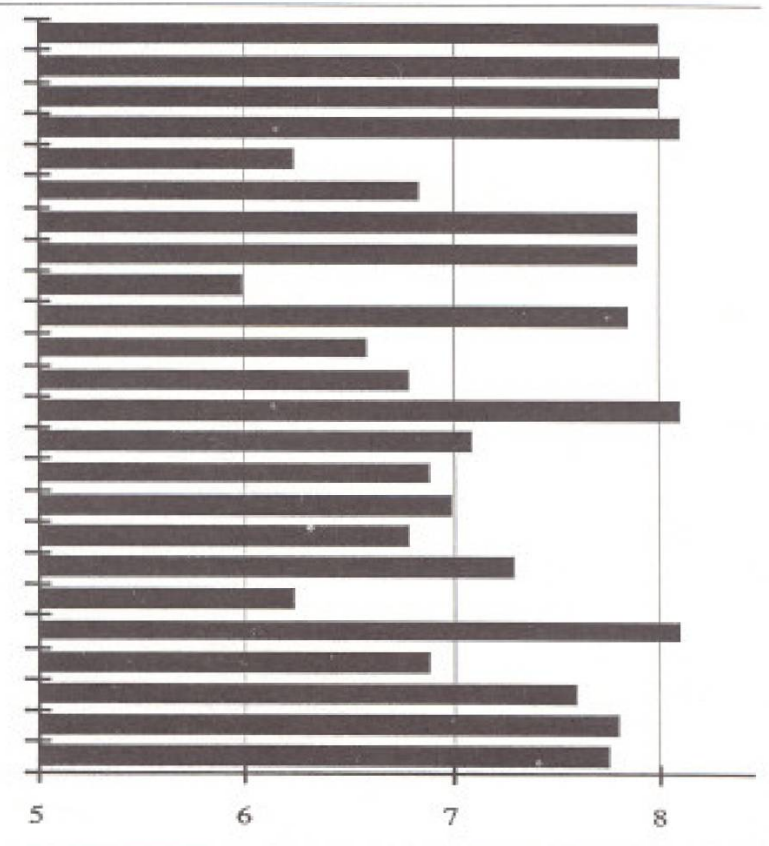

Source: Secor, 1989. 
Serait-ce le résultat de l'image que véhiculent certains journaux à sensation sur Montréal à travers le Québec?

\section{Les perceptions des Canadiens (vivant à l'extérieur du Québec)}

Le climat politique est un élément que les touristes vontgénéralement considérercomme critère de sélection au moment d'opter pour une destination. Il peut à lui seul donner une image négative à ladite destination. Peutêtre que l'après-Lac Meech influence la perception de Montréal qu'ont les Canadiens vivant à l'extérieur du Québec mais pour l'instant aucune étude ne vient confirmer ce fait et les Canadiens semblent attribuer une image positive à Montréal malgré tous ces événements. Notons que la majorité des touristes canadiens venant visiter Montréal sont originaires de $1^{\dagger}$ Ontario $(65,5 \%)$.

En général, les Canadiens considèrent Montréal comme une ville d'ambiance où il fait bon se divertir grâce à la variété de choses à voir et à faire (cf. tableau 6).

La culture française et les activités culturelles de la ville sont très bien perçues par les visiteurscanadiens. Lavie nocturne, laqualité de l'hébergement et de la restauration, le magasinageainsique l'accueiletl'hospitalité sont tous des éléments qui sont également trè̀s bien perçus.

Parcontre, Montréal semble décevoir parses coûts élevés d'hébergement et de restauration.

\section{Les perceptions des touristes d'outre-mer}

Très peu de données existent concernant la perception qu'ont ces touristes de Montréal.

Néanmoins, la ville semble posséder une image positive pour ces gens d'outre-merqui sont en majorité composés d'Européens de l'Ouest $(70,2 \%$ des visiteurs d'outre-mer à Montréal). Devant cette clientèle, Montréal n'a pas d'image de marque vraiment définie, L'aspect culturel de la ville, fondée d'abord sur sonfaitfrançais, ne semble pas constituer l'attraitprincipal. On perçoitplutôt Montréal comme un endroit où il fait bon vivre en toute sécurité et où l'accueil et l'hospitalité sont omniprésents. Montréal c'est aussi la ville pour magasiner et faire la fêtte. Elle est appréciée pour la qualité de ses restaurants et
TABLEAU 6

Mesures attitudinales des Canadiens face à Montréal

Les critères pour lesquels on observe une moyenne de plus de 7,5 peuvent être considérés comme des axes de force de Montréal.

Facilité pour se rendre Activités culturelles

Variété de choses à voir Variété de choses à faire

Propreté

Attraits touristiques

Vie nocturne

Qualité de l'hébergement

Coût de l'hébergement

Qualité des restaurants

Coûts de la restauration

Sécurité

Magasinage

Choses à faire (enfants)

Accueil, hospitalité

Rapport qualité/prix

Tempirature/climat

Information sur place

Attrait/culture française

Efficacité du transport local

Signalisation routière

Attrait général de Montréal

Popularité de Montréal

Ville pour faire la fête

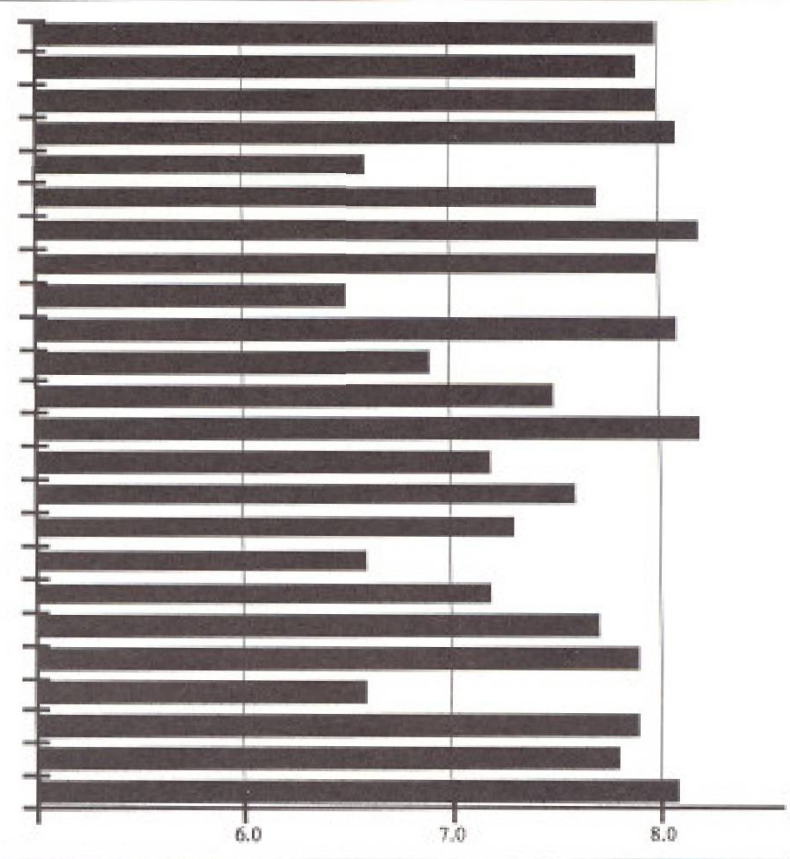

Source: Secor, 1989.

TABLEAU 7

Mesures attitudinales des touristes d'outre-mer face à Montréal

Les critères pour lesquels on observe une moyenne de plus de 7,5 peuvent être considérés comme des axes de force de Montréal

Facilité pour se rendre Activités culturelles

Variété de choses à voir

Variété de choses à faire

Propreté

Attraits touristiques

Vie nocturne

Qualité de 1'hébergement

Coût de l'hébergement

Qualitế des restaurants

Coûts de la restauration

Sécurité

Magasinage

Choses à faire (enfants)

Accueil, hospitalite

Rapport qualité/prix

Température/climat

Information sur place

Attrait/culture française

Efficacité du transport local

Signalisation routière

Attrait général de Montréal

Popularité de Montréal

Ville pour faire la fête

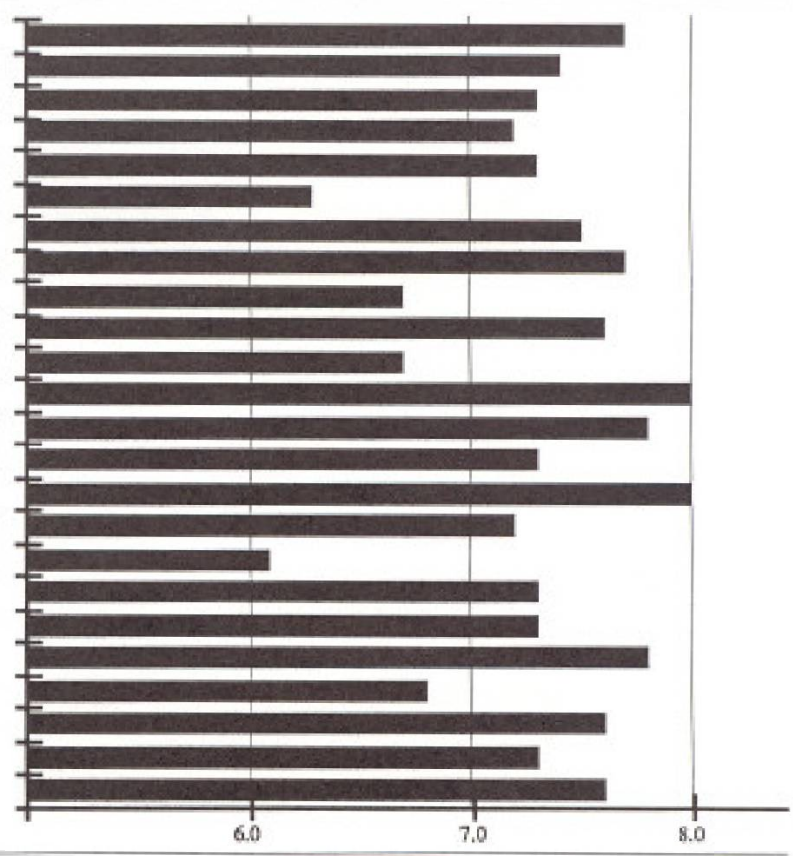

Source: Secor, 1989. 
de ses services d'hébergement. Par contre, les coûts qu'occasionnent ces services sont jugés élevés (cf. tableau 7).

Montréal, ville d'ambiance souvent considérée comme étant la porte d'entrée sécuritaireen Amérique duNord, voilà en quelques mots l'image qu'elle reflète sur les marchés. d'outre-mer.

Notons toutefois que pour le non-visiteur Montréal est souvent perçue comme le sont le Québec et le Canada, c'est-à-dire comme des grands espaces, occupés par les indiens.

\section{Conclusion}

Cette analyse fait ressortir que Montréal possède aujourd'hui une image d'ensemble qui luiest propre. Elle constitue dès le départ une force d'attraction pour le touriste.

Néanmoins, chacune desclientèlestraditionnelles de la ville accorde plus ou moins d'importance a l'un ou l'autre des attributs sur lesquels s'est forgé son image. Par exemple, les Québécois perçoivent Montréal comme étant une ville d'ambiance, les Américains: une ville propreet sécuritaire où l'histoire est au rendez-vous. Quant à la clientèle d'outre-mer, elle perçoit en Montréal une ville où l'accueil et l' hospitalité sont omniprésents.

Ces constatations nous indiquent que l'image de Montréal se doit d'être soutenue et en= tretenue par des stratégies marketing (ce qui comprend des stratégiees de gestion de la perception) qui doivent faire ressortir les forces d'attractions spécifiques à chacune des clientèles traditionnelles.

Il est important de positionner l'image de Montréal, faceà ses divers marchés, de façon àce que la ville soit perçue comme étant une destination pouvant satisfaire les besoins et intérêts de chacun de ses segments.

Pour ce faire, il est impératif que tous les intervenants aient une vision commune des stratégies à utiliser.

En effet, la concertation est nécessaire pour que Montréal en arrive à projeter une image claire, précise et surtout adéquate pour tous les marchés visés.
Un organisme comme l'Office des congrès et du tourisme du Grand Montréal pourrait très bien assurer le leadership nécessaire à une bonne gestion del'image de lamétropole.

\section{Bibliographie}

CLUZEAU, Patrick, Le Quebec touristique: indicatours sur les marehbs et sur les secteurs touristiques de 1980 à 1988, ministère du Tourisme du Québec, 1989, 587 pages.

GOUNERNEMENT DU CANADA, Industrie, Sciences et Technologie Canada, Longwoods Travel USA, Le marché américain des voyages d'agrément $1989,1991,90$ pages.

IMPACT RECHERCHE MARKETING ET COMMUNICA. TION, Étude de perception des Américains de Boston ot New York par rapport au Qubboc comme destination de vacances, 1990, 71 pages,

OFFICE DE PLANIFICATIONET DE DEVELOPPEMENT DU QUÉBEC, Latourisme à Montróal, 1986, 256 pages.

OFFICE DES CONGRES ET DU TOURISME DU GRAND MONTAEA.L. Analyse des coupures de la Presse américaine, 1990, 9 pages.

SANGUIN, Andre-Louis, Le tourisme a Montral, Departement degegographie, Universite du Quebeca Montrúal, 1985, 20 pages.

SECORINC. Etude ducompertement et de l'attl tude des differents segments de clientales touristiques a Montréal, 1989 , 189 pages.

VILLE DE MONTAEAL, CIDEM-Tourisme, Le tourisme à Montróal: recueil de données 1980-1987, 1987 . 603 pages:

VLLE DE MONTREAL, Pour la relanee du tourisme à Montroal, janvier 1988,117 pages. 